Planetary Systems in the Universe - Observation, Formation and Evolution

Proceedings IAU Symposium No. 202, (C)2004 IAU

Alan Penny, Pawel Artymowicz, Anne-Marie Lagrange, \& Sara Russell, eds.

\title{
Observations of Binary Systems Found in Numerical Simulations
}

\author{
Andrew F. Nelson \\ Max Planck Institute for Astronomy, Königstuhl 17, D-69251 \\ Heidelberg, Germany
}

\begin{abstract}
I present a two dimensional hydrodynamic simulation of a binary system consisting of two stars, each with a circumstellar disk, using a Smoothed Particle Hydrodynamic (SPH) code. I model the luminous output of this system in various millimeter and sub-millimeter wavelength bands appropriate for several new and soon to be on line interferometric telescopes and find that spiral structure present in the density structure of the disks will be difficult or impossible to observe in the observed emission from the system, due to the fact that disk photosphere temperatures are not very sensitive to the underlying density distribution.
\end{abstract}

\section{Initial Conditions and Physical Model}

The initial conditions for the simulation presented here are chosen to be quite similar to those observed (Rodriguez et al., 1998) in the L1551 IRS 5 binary system, and to those of other observed disk systems. Two star+disk systems are set up in an orbit around each other with semi-major axis $a=50 \mathrm{AU}$, and eccentricity $e=0.3$. Each disk is initially $15 \mathrm{AU}$ in radius, has mass $M_{D}=0.05 M \odot$ and begins with the material in the disk in circular orbits around each star, whose mass is $M_{*}=0.5 M_{\odot}$. Additional information about the initial configuration of this system can be found in Nelson (2000).

The thermal energy generation and dissipation mechanisms are outlined in detail in Nelson et al. (2000). To summarize, The thermal energy dissipation is modeled by passive blackbody radiation from each point in the disk at each time. The blackbody temperature is defined by a vertical structure calculation used to determine the vertical $(\rho, T)$ distribution and Rosseland mean opacities from the literature. Thermal energy is generated from bulk mechanical energy via viscous processes and shocks, which are modeled using an artificial viscosity. The implementation is common to many hydrodynamic codes and consists of a term proportional to the divergence of the velocity (the 'bulk' term) and a term proportional to the velocity divergence squared (the von Neumann-Richtmyer term). In Nelson et al. (2000) we estimated that in terms of an equivalent 'alpha' model, the dissipation due to both terms is of order a few $\times 10^{-3}$. Passive heating due to radiation absorbed from other parts of the system is not considered. 


\section{Results}

The system was evolved for eight binary orbits $\left(T_{\mathrm{Bin}} \approx 350 \mathrm{yr}\right)$. Due to tight space restrictions on this contribution, I refer the reader to Nelson (2000) figure 1 for a figure showing the the morphology of the simulation as it was just before and after the fourth periapse passage of the binary. Before periapse, the two disks are smooth and exhibit no visible spiral structure. However, during and after periapse, each disk develops strong, two armed spiral structures due to the mutual tidal interactions of the binary components. Over the next half orbit the spiral structure decays to the smooth condition and the cycle repeats as the components again approach each other.

Using a simple radiative cooling model allows the physical properties of the simulation to be related to quantities that observers at telescopes can measure. In this work, I extend the work of Nelson et al. (2000) on modeling the SEDs of the simulations to making synthetic images of the simulations in various wavelength bands.

At some time, the blackbody temperature of each SPH particle is mapped onto a $1024^{2}$ grid, corresponding to the region $\pm 75 \mathrm{AU}$ from the center of mass in both the $x$ and $y$ directions. The blackbody flux emitted from each grid zone at a given wavelength is then calculated and the result is convolved with a Gaussian point spread function, in order to simulate observation through a telescope of finite aperture. Although the two stars do not contribute to the energy input or output of the disks, except through their gravitational contribution, they are included in the post processed imaging calculation. Each star is assumed to be radiating as a blackbody with temperature $T_{*}=4300 \mathrm{~K}$ and luminosity $L_{*}=4 L_{\odot}$, appropriate for a young $\left(\sim 10^{5} \mathrm{yr}\right)$, half solar mass star.

In Fig. 1, synthesized images at four wavelengths are shown. In comparison to the very strong spiral structure present in the mass distribution, there is relatively little structure in the contours seen in the image, especially in the two bottom panels with wavelengths shortward of $1 \mathrm{~mm}$. Little if any of the internal density structure can be seen in the emitted radiation. A small amount of residual structure is visible, primarily due to the non-circular shape of the disks themselves.

The convolution with the PSF is not responsible for obscuring spiral structure which might otherwise be present in the image. The unconvolved images also lack a significant signature of spiral structure. Instead, the reason for the absence of structure is that the disk photosphere temperatures are not strongly sensitive to the underlying density structure because the disks are optically thick and only a thin surface layer contributes to the observed luminous output.

Both the luminosity at each wavelength in Fig. 1 and the total integrated over all wavelengths does not reproduce that of L1551 IRS 5. In each case, a smaller luminosity is derived from the simulation than that observed in L1551 IRS 5. Such a discrepancy is to be expected because the inventory of heating sources included in the model is not complete. Additional luminosity will be present due to contributions from the inner inost portion of the accretion disk not included in this simulation and from passive heating of the disks by circumstellar material. Including such heating sources will tend to increase the total luminosity and redistribute energy into the longer wavelengths, so that the discrepancy is reduced. 


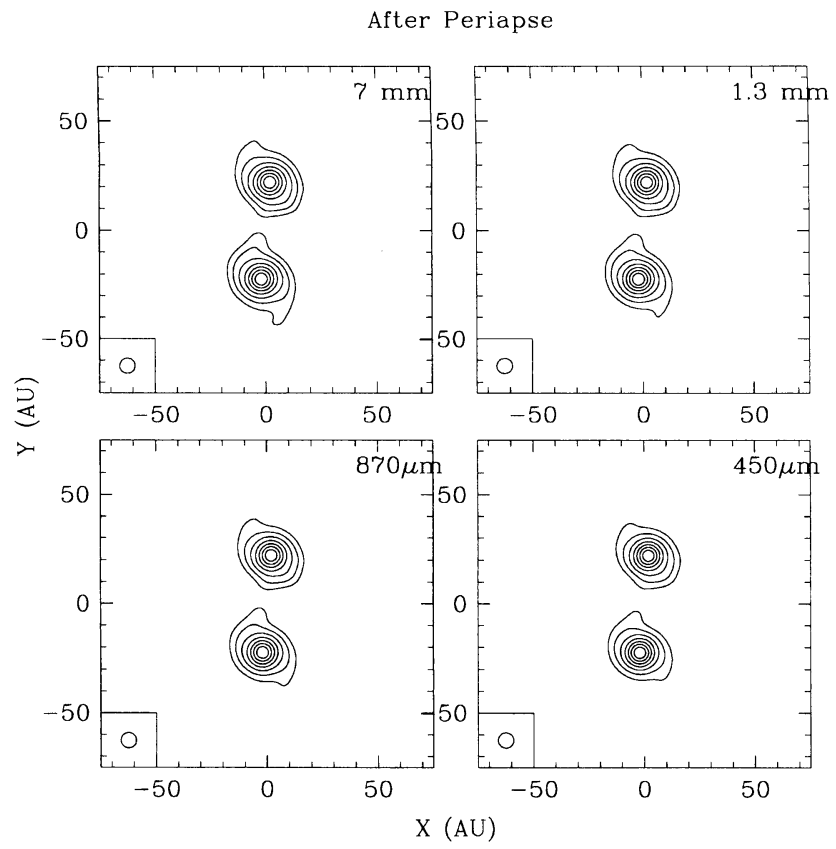

Figure 1. Synthesized images of the disk shown in Rodriguez et al. (1998) figure 1 (bottom) at four wavelengths. The image is convolved with a Gaussian point spread function (PSF) with $\mathrm{FWHM}=7 \mathrm{AU}$ (at the lower left in each panel) in order to simulate the view through a telescope of finite aperture. There are 8 contours in each panel and their spacing is a linear multiple of $.62,17.4,38.1$ and $134.6 \mathrm{mJy} /$ beam for the $7 \mathrm{~mm}, 1.3 \mathrm{~mm}, 870 \mu \mathrm{m}$ and $450 \mu \mathrm{m}$ panels, respectively.

\section{Concluding Remarks}

The main conclusion of this contribution is that spiral structure present in the density structure of an accretion disk will not be visible in the luminous output of optically thick accretion disks at wavelengths shorter than $1 \mathrm{~mm}$. Radiation from optically thin regions sensitive to volume density may still show evidence of spiral structure, however tests of this sort have not been done in the present work.

\section{References}

Rodriguez, L. F., D’Alessio, P., Willner, D. J., Ho, P. T. P., Torrelles, J. M., Curiel, S., Gómez, Y., Lizano, S., Pedlar, A., Cantó J., \& Raga, A. C. 1998, Nature, 395, 355

Nelson, A. F., Benz, W., \& Ruzmaikina, T. V. 2000, ApJ, 529, 357

Nelson, A. F. 2000, ApJ, 537, L66 